PHYSICAL REVIEW D 95, 019903(E) (2017)

\title{
Erratum: Kaon-nucleon scattering to one-loop order in heavy baryon chiral perturbation theory [Phys. Rev. D 92, 114033 (2015)]
}

Bo-Lin Huang and Yun-De Li

(Received 20 December 2016; published 17 January 2017)

DOI: $10.1103 /$ PhysRevD.95.019903

There were a number of errors in our paper. Equation (10) should take the following form:

$$
T_{K N, \bar{K} N}^{(I)}=\left(\frac{E_{N}+M_{N}}{2 M_{N}}\right)\left\{V_{K N, \bar{K} N}^{(I)}(q)+i \boldsymbol{\sigma} \cdot\left(\boldsymbol{q}^{\prime} \times \boldsymbol{q}\right) W_{K N, \bar{K} N}^{(I)}(q)\right\},
$$

then, Eqs. (12), (14), (20), (22), (29), and (31) should have their signs changed. The $C_{1}$ and $C_{2}$ of Eq. (36) should also have their signs changed. The $C_{3}$ and $C_{6}$ of Eq. (36) are replaced by $C_{3}=4 b_{10}+b_{11}$ and $C_{6}=-4 b_{9}-b_{11}$, respectively.

In Appendix A, Eq. (A2) should have its sign changed to be $Q^{2}=2 q^{2}(z-1)$. Equations (A5), (A7), (A14), (A16), (A31), (A33), (A72), and (A74) should also have their signs changed. $\alpha_{K}^{D F}, \alpha_{\eta}^{D F}, \beta_{K}^{D F}, \beta_{\eta}^{D F}, \delta_{K}^{D F}$, and $\delta_{\eta}^{D F}$ of Eq. (A12) should be given by

$$
\begin{aligned}
& \alpha_{K}^{D F}=D^{4}-30 D^{2} F^{2}+45 F^{4}, \\
& \alpha_{\eta}^{D F}=-D^{4}+9 D^{3} F-15 D^{2} F^{2}-9 D F^{3}, \\
& \beta_{K}^{D F}=-7 D^{4}+18 D^{2} F^{2}-27 F^{4}, \\
& \beta_{\eta}^{D F}=-5 D^{4}+21 D^{3} F-27 D^{2} F^{2}+27 D F^{3}, \\
& \delta_{K}^{D F}=5 D^{4}-54 D^{2} F^{2}+81 F^{4}, \\
& \delta_{\eta}^{D F}=D^{4}+3 D^{3} F-9 D^{2} F^{2}-27 D F^{3} .
\end{aligned}
$$

The terms $w^{3} \ln \frac{m_{K}}{\lambda}, w^{3} \ln \frac{m_{\pi}}{\lambda}$, and $w^{3} \ln \frac{m_{\eta}}{\lambda}$ should be multiplied by 2 in Eqs. (A21), (A23), (A25), and (A27). The terms $+\left(6 w m_{\pi}^{2}-2 w^{3}\right) \ln \frac{m_{\pi}}{\lambda},+\left(6 w m_{K}^{2}-2 w^{3}\right) \ln \frac{m_{K}}{\lambda}$, and $+\left(6 w m_{\eta}^{2}-2 w^{3}\right) \ln \frac{m_{\eta}}{\lambda}$ of Eqs. (A21), (A23), (A25), and (A27) should be replaced by $-2 w\left(m_{\pi}^{2}+w^{2} \ln \frac{m_{\pi}}{\lambda}\right),-2 w\left(m_{K}^{2}+w^{2} \ln \frac{m_{K}}{\lambda}\right)$, and $-2 w\left(m_{\eta}^{2}+w^{2} \ln \frac{m_{\eta}}{\lambda}\right)$, respectively. Equation (A22) should be given by

$$
\begin{aligned}
W_{K N}^{(1)}= & \frac{1}{288 \pi^{2} w^{2} f_{K}^{4}}\left\{-\alpha_{\pi}^{D F}\left[w^{3}+\pi m_{\pi}^{3}-2 w\left(m_{\pi}^{2}+w^{2} \ln \frac{m_{\pi}}{\lambda}\right)-2\left(w^{2}-m_{\pi}^{2}\right)^{3 / 2} \ln \frac{w+\sqrt{w^{2}-m_{\pi}^{2}}}{m_{\pi}}\right]\right. \\
& -\alpha_{K, 1}^{D F}\left[w^{3}+\pi m_{K}^{3}-2 w\left(m_{K}^{2}+w^{2} \ln \frac{m_{K}}{\lambda}\right)-2\left(w^{2}-m_{K}^{2}\right)^{3 / 2} \ln \frac{w+\sqrt{w^{2}-m_{K}^{2}}}{m_{K}}\right] \\
& +\alpha_{K, 2}^{D F}\left[\pi m_{K}^{3}+2 w m_{K}^{2}-w^{3}+2 w^{3} \ln \frac{m_{K}}{\lambda}-2\left(w^{2}-m_{K}^{2}\right)^{3 / 2}\left(i \pi-\ln \frac{w+\sqrt{w^{2}-m_{K}^{2}}}{m_{K}}\right)\right] \\
& -\alpha_{\eta}^{D F}\left[w^{3}+\pi m_{\eta}^{3}-2 w\left(m_{\eta}^{2}+w^{2} \ln \frac{m_{\eta}}{\lambda}\right)-2\left(m_{\eta}^{2}-w^{2}\right)^{3 / 2}\left(\arccos \frac{w}{m_{\eta}}\right) \theta\left(m_{\eta}^{2}-w^{2}\right)\right. \\
& \left.\left.-2\left(w^{2}-m_{\eta}^{2}\right)^{3 / 2}\left(\ln \frac{w+\sqrt{w^{2}-m_{\eta}^{2}}}{m_{\eta}}\right) \theta\left(w^{2}-m_{\eta}^{2}\right)\right]\right\} .
\end{aligned}
$$


Equation (A24) should be given by

$$
\begin{aligned}
W_{K N}^{(0)}= & \frac{1}{288 \pi^{2} w^{2} f_{K}^{4}}\left\{-\beta_{\pi}^{D F}\left[w^{3}+\pi m_{\pi}^{3}-2 w\left(m_{\pi}^{2}+w^{2} \ln \frac{m_{\pi}}{\lambda}\right)-2\left(w^{2}-m_{\pi}^{2}\right)^{3 / 2} \ln \frac{w+\sqrt{w^{2}-m_{\pi}^{2}}}{m_{\pi}}\right]\right. \\
& -\beta_{K, 1}^{D F}\left[w^{3}+\pi m_{K}^{3}-2 w\left(m_{K}^{2}+w^{2} \ln \frac{m_{K}}{\lambda}\right)-2\left(w^{2}-m_{K}^{2}\right)^{3 / 2} \ln \frac{w+\sqrt{w^{2}-m_{K}^{2}}}{m_{K}}\right] \\
& +\beta_{K, 2}^{D F}\left[\pi m_{K}^{3}+2 w m_{K}^{2}-w^{3}+2 w^{3} \ln \frac{m_{K}}{\lambda}-2\left(w^{2}-m_{K}^{2}\right)^{3 / 2}\left(i \pi-\ln \frac{w+\sqrt{w^{2}-m_{K}^{2}}}{m_{K}}\right)\right] \\
& -\beta_{\eta}^{D F}\left[w^{3}+\pi m_{\eta}^{3}-2 w\left(m_{\eta}^{2}+w^{2} \ln \frac{m_{\eta}}{\lambda}\right)-2\left(m_{\eta}^{2}-w^{2}\right)^{3 / 2}\left(\arccos \frac{w}{m_{\eta}}\right) \theta\left(m_{\eta}^{2}-w^{2}\right)\right. \\
& \left.\left.-2\left(w^{2}-m_{\eta}^{2}\right)^{3 / 2}\left(\frac{w+\sqrt{w^{2}-m_{\eta}^{2}}}{m_{\eta}}\right) \theta\left(w^{2}-m_{\eta}^{2}\right)\right]\right\} .
\end{aligned}
$$

Equation (A26) should be given by

$$
\begin{aligned}
W_{\bar{K} N}^{(1)}= & -\frac{1}{576 \pi^{2} w^{2} f_{K}^{4}}\left\{\gamma_{\pi}^{D F}\left[\pi m_{\pi}^{3}+2 w m_{\pi}^{2}-w^{3}+2 w^{3} \ln \frac{m_{\pi}}{\lambda}-2\left(w^{2}-m_{\pi}^{2}\right)^{3 / 2}\left(i \pi-\ln \frac{w+\sqrt{w^{2}-m_{\pi}^{2}}}{m_{\pi}}\right)\right]\right. \\
& +\gamma_{K, 1}^{D F}\left[\pi m_{K}^{3}+2 w m_{K}^{2}-w^{3}+2 w^{3} \ln \frac{m_{K}}{\lambda}-2\left(w^{2}-m_{K}^{2}\right)^{3 / 2}\left(i \pi-\ln \frac{w+\sqrt{w^{2}-m_{K}^{2}}}{m_{K}}\right)\right] \\
& -\gamma_{K, 2}^{D F}\left[w^{3}+\pi m_{K}^{3}-2 w\left(m_{K}^{2}+w^{2} \ln \frac{m_{K}}{\lambda}\right)-2\left(w^{2}-m_{K}^{2}\right)^{3 / 2} \ln \frac{w+\sqrt{w^{2}-m_{K}^{2}}}{m_{K}}\right] \\
& +\gamma_{\eta}^{D F}\left[\pi m_{\eta}^{3}+2 w m_{\eta}^{2}-w^{3}+2 w^{3} \ln \frac{m_{\eta}}{\lambda}-2\left(m_{\eta}^{2}-w^{2}\right)^{3 / 2}\left(\arccos \frac{-w}{m_{\eta}}\right) \theta\left(m_{\eta}^{2}-w^{2}\right)\right. \\
& \left.\left.-2\left(w^{2}-m_{\eta}^{2}\right)^{3 / 2}\left(i \pi-\ln \frac{w+\sqrt{w^{2}-m_{\eta}^{2}}}{m_{\eta}}\right) \theta\left(w^{2}-m_{\eta}^{2}\right)\right]\right\} .
\end{aligned}
$$

Equation (A28) should be given by 

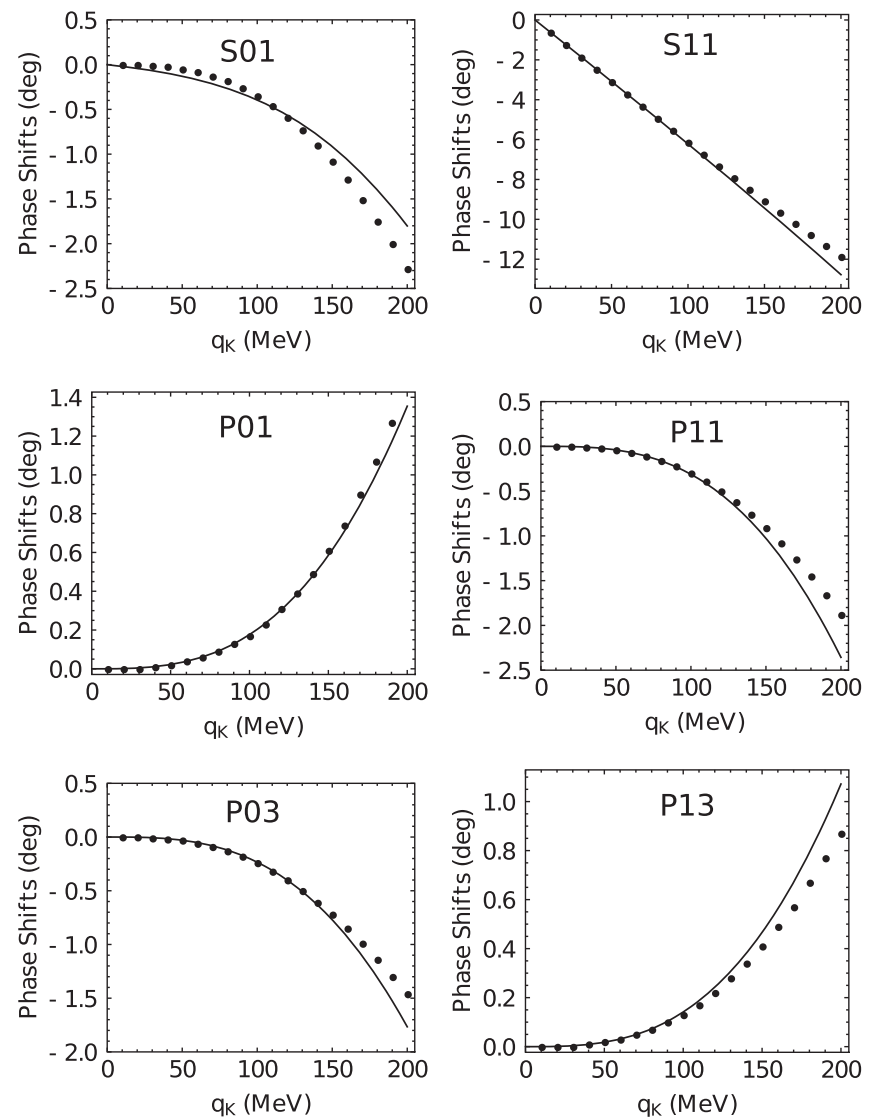

FIG. 1. Fits and predictions for the SP92 phase shifts versus the kaon laboratory momentum $q_{K}$ in $K N$ scattering. The fits in the S01, P01, and P03 waves are the data between 100 and $140 \mathrm{MeV}$ (solid dots), while the S11, P11, and P13 waves are between 50 and $90 \mathrm{MeV}$. For higher and lower energies, the phase shifts are predicted.

$$
\begin{aligned}
W_{\bar{K} N}^{(0)}= & -\frac{1}{576 \pi^{2} w^{2} f_{K}^{4}}\left\{\delta_{\pi}^{D F}\left[\pi m_{\pi}^{3}+2 w m_{\pi}^{2}-w^{3}+2 w^{3} \ln \frac{m_{\pi}}{\lambda}-2\left(w^{2}-m_{\pi}^{2}\right)^{3 / 2}\left(i \pi-\ln \frac{w+\sqrt{w^{2}-m_{\pi}^{2}}}{m_{\pi}}\right]\right]\right. \\
& +\delta_{K, 1}^{D F}\left[\pi m_{K}^{3}+2 w m_{K}^{2}-w^{3}+2 w^{3} \ln \frac{m_{K}}{\lambda}-2\left(w^{2}-m_{K}^{2}\right)^{3 / 2}\left(i \pi-\ln \frac{\left.\left.w+\sqrt{w^{2}-m_{K}^{2}}\right)\right]}{m_{K}}\right)\right] \\
& -\delta_{K, 2}^{D F}\left[w^{3}+\pi m_{K}^{3}-2 w\left(m_{K}^{2}+w^{2} \ln \frac{m_{K}}{\lambda}\right)-2\left(w^{2}-m_{K}^{2}\right)^{3 / 2} \ln \frac{w+\sqrt{w^{2}-m_{K}^{2}}}{m_{K}}\right] \\
& +\delta_{\eta}^{D F}\left[\pi m_{\eta}^{3}+2 w m_{\eta}^{2}-w^{3}+2 w^{3} \ln \frac{m_{\eta}}{\lambda}-2\left(m_{\eta}^{2}-w^{2}\right)^{3 / 2}\left(\arccos \frac{-w}{m_{\eta}}\right) \theta\left(m_{\eta}^{2}-w^{2}\right)\right. \\
& \left.-2\left(w^{2}-m_{\eta}^{2}\right)^{3 / 2}\left(i \pi-\ln \frac{w+\sqrt{w^{2}-m_{\eta}^{2}}}{m_{\eta}}\right) \theta\left(w^{2}-m_{\eta}^{2}\right)\right]
\end{aligned}
$$

$\alpha_{\pi}^{D F}, \alpha_{\eta}^{D F}, \beta_{\pi}^{D F}, \beta_{K, 1}^{D F}$, and $\beta_{\eta}^{D F}$ of Eq. (A29) should have their signs changed. $\alpha_{K, 1}^{D F}$ and $\delta_{\pi}^{D F}$ of Eq. (A29) should be given by

$$
\begin{aligned}
& \alpha_{K, 1}^{D F}=-13 D^{4}+42 D^{2} F^{2}-45 F^{4}, \\
& \delta_{\pi}^{D F}=27 D^{4}-54 D^{2} F^{2}+27 F^{4} .
\end{aligned}
$$



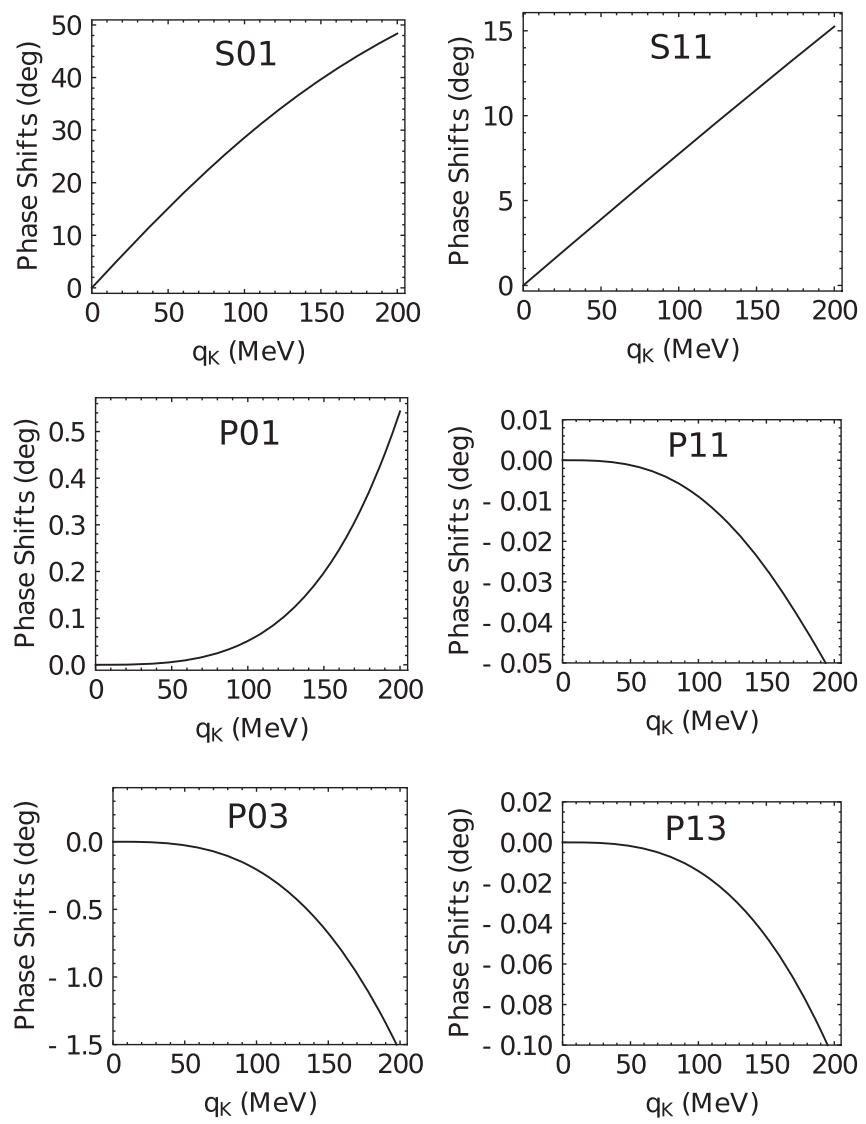

FIG. 2. The antikaon-nucleon $(\bar{K} N)$ phase shifts versus the kaon laboratory momentum $q_{K}$. The solid lines denote our predictions through the LECs determined by the $K N$ phase shifts and the corresponding data.

$\alpha_{K}^{D F}, \alpha_{\eta}^{D F}, \beta_{K}^{D F}, \beta_{\eta}^{D F}, \delta_{\pi}^{D F}, \delta_{K}^{D F}$, and $\delta_{\eta}^{D F}$ of Eq. (A38) should be given by

$$
\begin{aligned}
& \alpha_{K}^{D F}=40 D^{4}-48 D^{3} F+144 D^{2} F^{2}+144 D F^{3}+360 F^{4}, \\
& \alpha_{\eta}^{D F}=16 D^{4}+48 D^{2} F^{2}, \\
& \beta_{K}^{D F}=104 D^{4}-240 D^{3} F+144 D^{2} F^{2}-432 D F^{3}-216 F^{4}, \\
& \beta_{\eta}^{D F}=32 D^{4}-96 D^{3} F \\
& \delta_{\pi}^{D F}=12 D^{4}+72 D^{3} F+108 D^{2} F^{2}, \\
& \delta_{K}^{D F}=4 D^{4}+24 D^{3} F+72 D^{2} F^{2}+216 D F^{3}+324 F^{4}, \\
& \delta_{\eta}^{D F}=4 D^{4}+24 D^{3} F+36 D^{2} F^{2} .
\end{aligned}
$$

The terms $\left[2 r\left(m_{\pi}\right)\left(\arctan \frac{1}{r\left(m_{\pi}\right)}\right) \theta\left(4 m_{\pi}^{2}-Q^{2}\right)+\left(r\left(m_{\pi}\right) \ln \left|\frac{1+r\left(m_{\pi}\right)}{1-r\left(m_{\pi}\right)}\right|-i \pi r\left(m_{\pi}\right)\right) \theta\left(Q^{2}-4 m_{\pi}^{2}\right)\right]$ and $r\left(m_{K}\right) \arctan \frac{1}{r\left(m_{K}\right)}$ should be replaced by $r\left(m_{\pi}\right) \ln \left|\frac{1+r\left(m_{\pi}\right)}{1-r\left(m_{\pi}\right)}\right|$ and $\frac{1}{2} r\left(m_{K}\right) \ln \left|\frac{1+r\left(m_{K}\right)}{1-r\left(m_{K}\right)}\right|$ in Eqs. (A58), (A59), (A60), and (A61), respectively. The terms $-12 \pi m_{\pi}^{2} Q^{2} K_{0}\left(m_{\pi}\right),-4 \pi m_{K}^{2} Q^{2} K_{0}\left(m_{K}\right),\left(8 m_{K}^{2}+3 Q^{2}\right),\left(15 m_{\pi}^{2}+21 m_{\eta}^{2}-18 m_{\pi} m_{\eta}+3 Q^{2}\right),\left(12 m_{\eta}^{4}-60 m_{\pi}^{2} m_{\eta}^{2}-18 m_{\pi}^{2} Q^{2}\right.$ $\left.-6 m_{\eta}^{2} Q^{2}+3 Q^{4}\right)$, and $\left(12 m_{\pi}^{4}-36 m_{\pi}^{2} m_{\eta}^{2}-18 m_{\eta}^{2} Q^{2}-6 m_{\pi}^{2} Q^{2}+3 Q^{4}-24 m_{\eta}^{4}\right)$ of Eqs. (A62), (A63), (A64), and (A65) should be replaced by $+36 \pi m_{\pi}^{2} Q^{2} K_{0}\left(m_{\pi}\right),+12 \pi m_{K}^{2} Q^{2} K_{0}\left(m_{K}\right),\left(8 m_{K}^{2}-9 Q^{2}\right),\left(15 m_{\pi}^{2}+21 m_{\eta}^{2}-18 m_{\pi} m_{\eta}-9 Q^{2}\right)$, $-\left(12 m_{\eta}^{4}-60 m_{\pi}^{2} m_{\eta}^{2}+30 m_{\pi}^{2} Q^{2}-6 m_{\eta}^{2} Q^{2}+3 Q^{4}\right)$, and $-\left(12 m_{\pi}^{4}-36 m_{\pi}^{2} m_{\eta}^{2}+30 m_{\eta}^{2} Q^{2}-6 m_{\pi}^{2} Q^{2}+3 Q^{4}-24 m_{\eta}^{4}\right)$, respectively. The remaining $Q^{2}$ 's of Eqs. (A62), (A63), (A64), and (A65) should have their signs changed. Equations (A66), (A67), (A68), and (A69) should be given by 

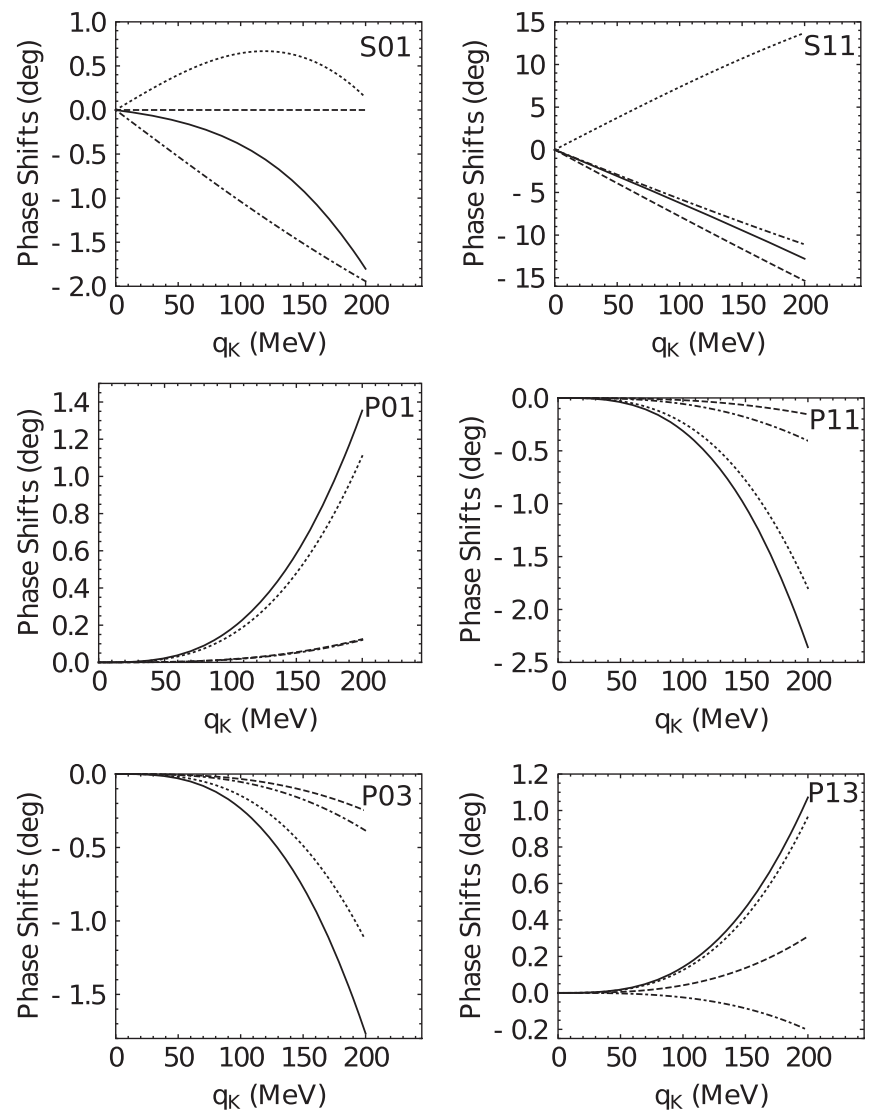

FIG. 3. Convergence properties as exemplified by our predictions for the $K N$ phase shifts. The dashed, dotted, and dashed-dotted lines denote the first, second, and third order, respectively. The solid lines give the sum of the first-, second-, and third-order contributions.

$$
\begin{gathered}
K_{0}(m)=-\frac{1}{8 \pi \sqrt{-Q^{2}}} \arctan \frac{\sqrt{-Q^{2}}}{2 m}, \\
H_{0}(m)=-m^{2}+\frac{Q^{2}}{6}-5 m^{2} \ln \frac{m}{\lambda}+\left(2 m^{2}+\frac{Q^{2}}{4}\right)\left[1-2 \ln \frac{m}{\lambda}-r(m) \ln \left|\frac{1+r(m)}{1-r(m)}\right|\right], \\
H\left(m_{1}, m_{2}\right)=\frac{1}{32 \pi^{2} \sqrt{-Q^{2}}}\left[F_{1}\left(y_{1}\right)+F_{1}\left(y_{2}\right)+F_{2}\left(x_{1}\right)+F_{2}\left(x_{2}\right)-F_{3}\left(z_{1}\right)-F_{3}\left(z_{2}\right)\right],
\end{gathered}
$$

and

$$
\begin{aligned}
F_{1}(x) & =\mathrm{Li}_{2}\left(\frac{z_{0}+2 Q^{2}}{z_{0}-2 \sqrt{-Q^{2}} x}\right)-\mathrm{Li}_{2}\left(\frac{z_{0}}{z_{0}-2 \sqrt{-Q^{2}} x}\right), \\
F_{2}(x) & =-\mathrm{Li}_{2}\left(\frac{z_{0}+2 Q^{2}}{z_{0}-2 \sqrt{-Q^{2}} x}\right)-\frac{1}{2} \ln ^{2}\left(\frac{z_{0}-2 \sqrt{-Q^{2}} x}{\lambda^{2}}\right), \\
F_{3}(x) & =-\mathrm{Li}_{2}\left(\frac{z_{0}}{z_{0}-2 \sqrt{-Q^{2}} x}\right)-\frac{1}{2} \ln ^{2}\left(\frac{z_{0}-2 \sqrt{-Q^{2}} x}{\lambda^{2}}\right), \\
z_{0} & =m_{2}^{2}-m_{1}^{2}-Q^{2}, \quad x_{1,2}=\sqrt{-Q^{2}} \pm i m_{2}, \quad z_{1,2}= \pm i m_{1}, \\
y_{1,2} & =-\frac{1}{2 \sqrt{-Q^{2}}}\left(m_{1}^{2}-m_{2}^{2}+Q^{2} \pm \sqrt{\left[Q^{2}-\left(m_{1}+m_{2}\right)^{2}\right]\left[Q^{2}-\left(m_{1}-m_{2}\right)^{2}\right]}\right),
\end{aligned}
$$

respectively. 
In the results and discussion, the discussion about the low-energy constant $C_{2}$ (in the second paragraph of the sixth page) should be deleted, and Fig. 4 should also be deleted. The statements "and successfully reduce one parameter" in the abstract and "and find a constraint among the LECs" in the summary should be deleted. Equations (42) and (43) about the constants should be given by

$$
\begin{aligned}
& C_{1}=1.99 \pm 0.11 \mathrm{GeV}^{-1}, \\
& C_{2}=-0.45 \pm 0.11 \mathrm{GeV}^{-1}, \\
& C_{3}=6.36 \pm 0.09 \mathrm{GeV}^{-1},
\end{aligned}
$$

with $\chi^{2} /$ d.o.f. $\sim 0.91$ and

$$
\begin{aligned}
& C_{4}=3.01 \pm 0.21 \mathrm{GeV}^{-1}, \\
& C_{5}=-5.10 \pm 0.21 \mathrm{GeV}^{-1}, \\
& C_{6}=-5.13 \pm 0.12 \mathrm{GeV}^{-1},
\end{aligned}
$$

with $\chi^{2} /$ d.o.f. $\sim 2.49$. The statement "One can find that the uncertainty of the parameter $C_{2}$ is very large when compared to the $C_{2}$ value. For this, a more detailed discussion is given below." in the last paragraph of the fifth page should be deleted. Figure 3 on the sixth page should be replaced by the following Fig. 1. On the sixth page the first sentence below Eq. (44) should be replaced by "The LEC combination $C_{1}+C_{2}$ determined by the phase shifts from Eq. (13) is $1.54 \mathrm{GeV}^{-1}$, while the $C_{4}+C_{5}$ from Eq. (14) is $-2.09 \mathrm{GeV}^{-1}$." Figure 5 on the seventh page should be replaced by the following Fig. 2. $a_{K N}^{(0)}$, $a_{\bar{K} N}^{(1)}$, and $a_{\bar{K} N}^{(0)}$ should be $-0.01,0.41$, and 1.63 in "Prediction A" of Table I, respectively. Figure 6 on the eighth page should be replaced by the following Fig. 3. The changed formulas result in these changed constants, figures, and statements; the other main statements are not changed. These corrections are all fully incorporated into the paper arXiv:1612.06163. 